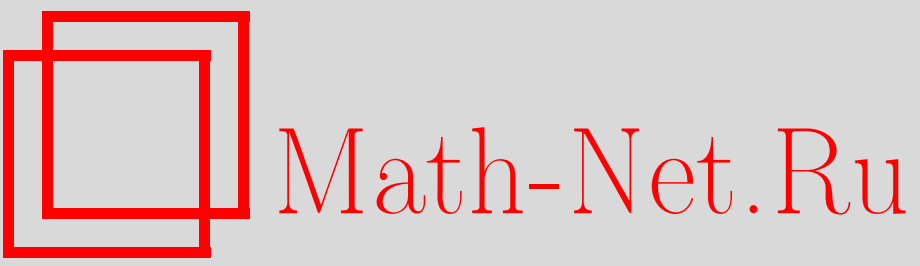

Ю. П. Вирченко, О. И. Шеремет, Геометрические модели статистической теории фрагментации, ТМФ, 2001, том 128, номер 2, 161-177

DOI: https://doi.org/10.4213/tmf489

Использование Общероссийского математического портала Math-Net.Ru подразумевает, что вы прочитали и согласны с пользовательским соглашением

http://www.mathnet.ru/rus/agreement

Параметры загрузки:

IP: 18.234 .197 .8

26 апреля 2023 г., 05:16:28 
ТЕОРЕТИЧЕСКАЯ

И МАТЕМАТИЧЕСКАЯ

ФИЗИКА

Том 128, № 2

август, 2001

(c) 2001 r.

Ю.П. Вирченко*, О.И. Шеремет*

\title{
ГЕОМЕТРИЧЕСКИЕ МОДЕЛИ СТАТИСТИЧЕСКОЙ ТЕОРИИ ФРАГМЕНТАЦИИ
}

\begin{abstract}
Предложен подход к описанию процесса фрагментации среды на основе изучения стохастической геометрии состояний этой среды. Такой подход позволяет учитывать взаимное влияние фрагментов на процесс фрагментации, связанное с их расположением друг относительно друга и, в частности, учитывать размерность дробящегося объекта. В рамках этого подхода анализируется одномерная модель - стохастический процесс с дискретным временем и с фазовым пространством, состоящим из разбиений на фрагменты действительной оси. Выведено управляющее уравнение для функции распределения по размерам и доказано существование предельного закона распределения.
\end{abstract}

\section{1. ВВЕДЕНИЕ}

Статистическая теория фрагментации занимается проблемой описания разнообразных процессов разрушения на основе вероятностно-феноменологического подхода. Она имеет широкий спектр приложений в теоретической физике при изучении явлений, происходящих в микро- и макромире (распад тяжелых атомных ядер, разрыв полимерных молекул, образование ландшафта планет под действием метеоритов и др.). Использовать традиционные методы теоретической физики для изучения подобных явлений невозможно, поскольку эти методы не приспособлены к описанию эволюции среды, находяшейся под влиянием большого количества неконтролируемых воздействий. В этих условиях основными становятся чисто вероятностные методы моделирования, связанные с понятиями независимости и равнораспределенности.

Одна из возможных постановок задач, ставшая теперь классической, была дана в работе [1], где предложена вероятностная схема каскадной фрагментации. В рамках этой схемы была доказана предельная теорема для распределения фрагментов по размерам при стремлении длительности эволюции системы к бесконечности.

Введенная в работе [1] модель основана на двух существенных предположениях о характере эволюционного процесса фрагментации.

Во-первых, предполагается, что дробление фрагментов происходит однородно во времени и осушествляется самоподобным образом. Это означает, что распределение веро-

${ }^{*}$ Институт монокристаллов НАН Украины, Харьков, Украина 
ятностей образования в каждом отдельном акте дробления осколков зависит только от их количества и отношения их размера к размеру разваливаюшегося фрагмента, но не от абсолютного размера (и геометрической формы) этого фрагмента. Последнее зачастую не имеет места в реальных физических процессах. Например, это предположение не учитывает так называемый масштабный әффект, известный в материаловедении и состоящий в том, что вероятность развала фрагмента растет вместе с его размером. Кроме того, в рамках этого предположения детали стохастического механизма, определяющего отдельные акты дробления, игнорируются.

Во-вторых, предполагается, что дробление каждого отдельного фрагмента после его образования не зависит от эволюции остальных фрагментов, а весь процесс в целом протекает каскадным образом. Этим самым не учитывается возможность коллективного поведения всей среды при дробящих воздействиях.

Первое предположение позволяет характеризовать фрагменты только одним параметром - их размером, а состояние среды - "числами заполнения" в пространстве размеров. Второе дает возможность моделировать эволюцию среды в виде ветвящегося марковского случайного прочесса со значениями в фазовом пространстве состояний, характеризуемых этими числами.

Большинство работ, посвященных изучению процессов фрагментации и проблеме их математического описания, основано на описанном подходе, хотя и с некоторыми видоизменениями. Учитывалось влияние возраста и размера фрагментов на отдельные акты дробления (см. работу [2], где выполнено довольно подробное исследование этого влияния). Варьировался характер стохастической динамики, однако без отказа от свойства марковости. Это, в частности, приводило к моделям, в которых появлялось взаимодействие фрагментов, например, вследствие определенного геометрического расположения осколков при распаде фрагмента. В последнем случае возникает различие между процессами фрагментации, протекаюшими в пространствах различной размерности. В частности, одномерные модели такого типа имеют название моделей случайного разрезания (scission model) [3], [4]. Общим принципом моделирования фрагментации во всех этих работах является составление феноменологического управляющего уравнения (master equation) для функции распределения по размерам с явным построением случайного процесса [1], [2], [4], [5]. Из последних работ, относяшихся к этому направлению, упомянем [6], [7].

Возможно, однако, вероятностное описание фрагментации среды на более глубоком уровне, основанное на введении стохастической динамики в терминах фазового пространства геометрических состояний всей среды в целом, т.е. в терминах совокупностей случайных множеств - фрагментов, ее составляюших. Разумеется, математическая реализация такого подхода сложна и сушественные проблемы возникают уже на стадии построения фазового пространства в многомерном случае. Однако, по нашему мнению, только в рамках такого подхода, которьй мы называем геометрическим, возможно последовательно учесть взаимное влияние фрагментов в процессе фрагментации. Отличие описанного выше традиционного подхода к проблемам фрагментации от геометрического примерно такое же, как отличие подходов в неравновесной статистической механике 
многих тел, основанных соответственно на феноменологическом составлении управляюших уравнений и на микроскопическом их выводе (см., например, [8]).

В этой работе мы анализируем одномерную геометрическую модель фрагментации стохастический процесс с дискретным временем и с фазовым пространством, состоящим из разбиений на фрагменты действительной оси. Основной целью работы является вывод управляющего уравнения для функции распределения по размерам и доказательство существования для этой функции предельного закона распределения. Этот закон уже не является универсальным и зависит от динамического механизма дробления. В разделе 2 описан общий принцип построения геометрических моделей фрагментации. С целью его демонстрации в разделе 3 мы анализируем элементарную одномерную модель дробления. В разделе 4 вводится одномерная геометрическая модель фрагментации каскадного типа, аналогичная рассмотренной в работе [1]. Разделы 5 и 6 посвящены доказательству предельной теоремы, которая является усиленным вариантом теоремы из статьи [9].

\section{2. СЛУЧАЙНЫЕ ТОЧЕЧНЫЕ ПОЛЯ И ЗАДАЧА ФРАГМЕНТАЦИИ}

$\Phi$ рагментацию среды будем описывать в терминах случайных множеств $\{\widetilde{X}\}$ в $\mathbb{R}^{d}$. В дальнейшем тильда над символом будет указывать на тот факт, что соответствуюший математический объект является случайным. Эти случайные множества являются $c e-$ парабельными [10], т.е. каждая их реализация восстанавливается с вероятностью 1 по ее пересечению с плотным в $\mathbb{R}^{d}$ множеством точек. Реализация $\widetilde{X}$ сепарабельного случайного множества распадается на связные компоненты, $\widetilde{X}=\bigcup_{i} \tilde{\sigma}_{i}$, где $\tilde{\sigma}_{i}-$ фрагменты среды, каждый из которых представляет собой компактную область в $\mathbb{R}^{d}$, ограниченную замкнутой непрерывной поверхностью.

При изучении фрагментации приходится моделировать различные физические ситуации. Среди них необходимо выделить группу таких, в которых развал объекта (например, атомного ядра) представляет собой однократное случайное событие. Такие события будем назьвать дроблением. При этом необходимо различать ситуации, когда число осколков невелико, как в указанном примере, и когда их количество настолько большое (например, при хрупком разрушении твердого тела под давлением), что изучение фрагментов одного такого события достаточно для получения достоверной информации об их статистических характеристиках посредством термодинамического предельного nерехода. Нас будет интересовать последний случай, как тесно примыкающий по постановке задачи к статистической механике.

Вторую большую группу типов фрагментации составляют те случаи, когда она представляет собой динамический процесс, который моделируется случайным проиессом $\left\{\widetilde{X}_{t}\right\}$ в математическом смысле. Такая ситуация рассматривалась в работе [1]. Именно ее мы и будем называть фрагментацией. Случайный процесс при этом можно вводить как с непрерьвным временем, так и с дискретным. Фазовое пространство этого процесса составляют точечные множества в $X_{0}$, где $X_{0}$ - большая область в $\mathbb{R}^{d}$. Заметим, что для каждой реализации случайного процесса $\widetilde{X}_{t} \subset \widetilde{X}_{s}$ при $t>s$. Обычно из-за того, что 
основной проблемой теории является изучение геометрии отдельных фрагментов, при конструировании конкретных моделей фрагментации игнорируют расположение этих фрагментов в $\mathbb{R}^{d}$. Тем не менее, наиболее последовательное описание динамики или однократного события дробления осушествляется в терминах случайных процессов с фазовым пространством $2^{X_{0}}$. Конструктивное задание случайных процессов такого рода сталкивается с трудностью математического описания их отдельных случайных реализаций в многомерном случае [11]. Поэтому в данной работе мы ограничиваемся одномерным случаем, когда описание стохастической геометрии фрагментов легко осуществляется посредством задания случайного процесса $\left\{\widetilde{Y}_{t}\right\}$ наборов границ фрагментов. Реализацией этого процесса может быть либо конечное случайное подмножество точек из $X_{0}$, либо счетное случайное подмножество в $\mathbb{R}$, представляюшее собой случайный поток событий. Для обоих случаев методы их вероятностного описания хорошо разработаны.

Отметим, наконец, что среди динамических процессов фрагментации особое значение имеют такие, в которых сохраняется вешество. Случайные процессы $\left\{\widetilde{X}_{t}\right\}$, моделируюшие такую ситуацию, обладают тем свойством, что mes $\widetilde{X}_{t}=$ const. В этой работе мы рассматриваем процесс именно такого типа.

\section{3. ДИСКРЕТНАЯ ОДНОМЕРНАЯ МОДЕЛЬ ДРОБЛЕНИЯ}

Рассмотрим простейшую модель дробления, основанную на последовательности независимых испытаний. Эта модель обладает тем достоинством, что основная задача теории - вычисление распределения фрагментов по размерам - допускает точное решение.

Пусть $\{\tilde{\xi}(n) ; n \in \mathbb{N}\}$ - последовательность независимых испытаний со значениями $\tilde{\xi}(n)=0,1$. Пусть $\mathbf{P}\{\xi(n)=1\}=1-q, 0<q<1$. Совокупность интервалов $\left(\tilde{r}_{i}, \tilde{r}_{i+1}\right)=\tilde{\rho}_{i}, \quad i \in \mathbb{N}$, моделирует фрагментацию “среды" $\mathbb{R}$, где $\left\{\tilde{r}_{i}\right\}$ - случайная последовательность, определенная формулой $\tilde{\xi}\left(\tilde{r}_{i}\right)=1$ и упорядоченная по возрастанию.

Построим случайную последовательность $\left\{\tilde{\mu}_{i} ; i \in \mathbb{N} \cup\{0\}\right\}, \tilde{\mu}_{i}=\tilde{r}_{i+1}-\tilde{r}_{i}$, размеров фрагментов. Эта последовательность является однородной последовательностью независимых испытаний со значениями в $\mathbb{N}$, распределенными согласно закону

$$
\mathbf{P}\left\{\tilde{\mu}_{i}=m\right\}=(1-q) q^{m-1} .
$$

В самом деле,

$$
\begin{aligned}
& \mathbf{P}\left\{\tilde{\mu}_{s}, \ldots, \tilde{\mu}_{1}, \tilde{\mu}_{0}\right\}=\mathbf{P}\left\{\tilde{r}_{s+1}-\tilde{r}_{s}=m_{s}, \ldots, \tilde{r}_{1}-\tilde{r}_{0}=m_{1}, \tilde{r}_{0}=m_{0}\right\}= \\
& \quad=\mathbf{P}\left\{\tilde{r}_{s+1}=m_{s}+\cdots+m_{0}, \ldots, \tilde{r}_{1}=m_{0}\right\}= \\
& \quad=\mathbf{P}\left\{\xi\left(m_{s}+\cdots+m_{0}\right)=1, \ldots, \xi\left(m_{0}\right)=1 ; \xi(l)=0, l \neq m_{0}, \ldots, m_{0}+\cdots+m_{s}\right\}= \\
& \quad=(1-q)^{s+1} q^{m_{s}+\cdots+m_{0}-s-1}=\mathbf{P}\left\{\tilde{\mu}_{s}=m_{s}\right\} \ldots \mathbf{P}\left\{\tilde{\mu}_{1}=m_{1}\right\} \mathbf{P}\left\{\tilde{\mu}_{0}=m_{0}\right\},
\end{aligned}
$$

так как испытания $\tilde{\xi}(n), n \in \mathbb{N}$, независимы в совокупности. 
Рассмотрим теперь произвольный конечный отрезок длины $N$ последовательности $\{\tilde{\xi}(n)\}$. Пусть таковым будет отрезок с $n=0,1,2, \ldots, N$. В этом отрезке выделим последовательность случайных величин - длин фрагментов $\tilde{\mu}_{0}^{(N)}=\tilde{r}_{1}, \tilde{\mu}_{1}^{(N)}=\tilde{r}_{2}-\tilde{r}_{1}, \ldots$, $\tilde{\mu}_{\tilde{\nu}-1}^{(N)}=\tilde{r}_{\tilde{\nu}}-\tilde{r}_{\tilde{\nu}-1}, \tilde{\mu}_{\tilde{\nu}}^{(N)}=N-\tilde{r}_{\tilde{\nu}}$. Здесь $\tilde{\nu}=\max \left\{i: \tilde{r}_{i} \in(1,2, \ldots, N)\right\}$. Обозначим $\tilde{\nu}_{l}^{(N)}=\operatorname{Card}\left\{i: \tilde{\mu}_{i}^{(N)}=l\right\}-$ случайное число фрагментов из этой последовательности, имеющих длину $l$. Необходимо найти закон распределения этого случайного числа в зависимости от $l$ при термодинамическом предельном переходе $N \rightarrow \infty$. Таким образом, мы будем интересоваться величиной

$$
\lim _{N \rightarrow \infty} \frac{\tilde{\nu}_{l}^{(N)}}{\tilde{\nu}^{(N)}} \equiv p_{l},
$$

где $\tilde{\nu}^{(N)}$ - полное число фрагментов на отрезке $(0, N)$. Так как $\sum_{l=1}^{\infty} \tilde{\nu}_{l}^{(N)}=\tilde{\nu}^{(N)}$, то $\sum_{l=1}^{\infty} p_{l}=1$. Число $\tilde{\nu}^{(N)}$ отличается не более чем на 1 от числа $\tilde{\nu}$ "успехов" в последовательности независимых испытаний $\{\tilde{\xi}(n) ; n=1, \ldots, N\}$, поэтому согласно предельной теореме Бернулли имеем почти наверное, что

$$
\lim _{N \rightarrow \infty} \frac{\tilde{\nu}^{(N)}}{N}=\lim _{N \rightarrow \infty} \frac{\left\langle\tilde{\nu}^{(N)}\right\rangle}{N}=1-q,
$$

т.е. с вероятностью $1 \lim _{N \rightarrow \infty} \tilde{\nu}^{(N)}=\infty$. С другой стороны, так как $\tilde{\nu}^{(N)}$ монотонно не убывает с увеличением $N$ и любая точка изменения одной из случайных величин $\tilde{\nu}_{l}^{(N)}$ с $l=1,2, \ldots$ при увеличении $N$ связана с точкой изменения величины $\tilde{\nu}^{(N)}$, то можно ввести случайную функцию $\tilde{\nu}_{l}(s)=\tilde{\nu}_{l}^{(N)}$ при $\tilde{\nu}^{(N)}=s$. В этом случае величина $(2)$ заменяется на

$$
\lim _{s \rightarrow \infty} \frac{\tilde{\nu}_{l}(s)}{s}=p_{l} .
$$

Величины $\left\{\tilde{\mu}_{i}\right\}$ независимы в совокупности. Случайная же последовательность $\left\{\tilde{\nu}_{l}(s)\right.$; $s=1,2, \ldots\}$ является “считающей” функцией $\tilde{\nu}_{l}(s)=\sum_{i=1}^{s} \delta_{\tilde{\mu}_{i} l}$ для однородной последовательности $\left\{\tilde{\mu}_{i} ; i=1,2, \ldots\right\}$ независимых испытаний. Как и в $(3)$, на основании теоремы Бернулли (свойство әргодичности последовательности $\left.\left\{\tilde{\mu}_{n}\right\}\right)$ и закона распределения (1) заключаем, что с вероятностью 1 имеет место соотношение

$$
\lim _{s \rightarrow \infty} \frac{\tilde{\nu}_{l}(s)}{s}=(1-q) q^{l-1}
$$

что позволяет с учетом (4) решить задачу о предельном распределении фрагментов по размерам. Заметим, наконец, что определение (2) распределения по размерам может быть заменено на

$$
\lim _{N \rightarrow \infty} \frac{\left\langle\tilde{\nu}_{l}^{(N)}\right\rangle}{\left\langle\tilde{\nu}^{(N)}\right\rangle} \equiv p_{l}
$$

ввиду выполнимости (3) и аналогичного соотношения

$$
\lim _{N \rightarrow \infty} \frac{\tilde{\nu}_{l}^{(N)}}{N}=\lim _{N \rightarrow \infty} \frac{\left\langle\tilde{\nu}_{l}^{(N)}\right\rangle}{N}
$$

с вероятностью 1. 


\section{4. СЛУЧАЙНЫЙ ПРОЦЕСС ФРАГМЕНТАЦИИ ОДНОМЕРНОЙ СРЕДЫ}

Построим явным образом случайный процесс $\left\{\widetilde{X}_{t}\right\}$ с дискретным временем $t \in \mathbb{N}_{0}=$ $\{0,1,2, \ldots\}$, который описывает последовательные стадии фрагментации на совокупность связных компонент одномерного образца $X_{0}$ - отрезка в $\mathbb{R}$. Значениями процесса $\tilde{X}_{t}$ являются подмножества $X_{0}$. Связные компоненты $\tilde{\sigma}_{i}(t), i=1, \ldots, \tilde{\nu}(t)$, каждого подмножества $\widetilde{X}_{t}$ будем назьвать фрагментами дробления. Множества $\widetilde{X}_{t}$ обладают свойством подчинения, указываюшим на то, что каждое семейство $\widetilde{X}_{t+1}$ является последующей стадией дробления материала по отношению к стадии дробления, описываемой семейством $\widetilde{X}_{t}$. А именно, необходимо, чтобы каждая связная компонента $\tilde{\sigma}_{j}(t+1)$, принадлежашая $\widetilde{X}_{t+1}$, являлась подмножеством для некоторого фрагмента $\tilde{\sigma}_{i}(t)$, входящего в семейство $\widetilde{X}_{t}$. При этом разность $\cup \tilde{\sigma}_{i}(t) \backslash \cup \tilde{\sigma}_{j}(t+1)$ по определению состоит лишь из конечного случайного набора точек, которые являются точками деления.

Будем считать, что $X_{0}=[0,1]$. С целью построения процесса $\widetilde{X}_{t}$ введем случайный процесс $\tilde{Y}_{t}$, который в каждый момент $t=0,1,2, \ldots$ представляет собой случайный конечный набор точек деления, содержащихся в $[0,1]$. Тогда процесс $\widetilde{X}_{t}$ индуцируется процессом $\widetilde{Y}_{t}$ по формуле

$$
\widetilde{X}_{t}=[0,1] \backslash \widetilde{Y}_{t} .
$$

Определим для каждого $t$ множество

$$
\mathfrak{A}_{t}=\left\{\frac{s}{N^{t}} ; s=0,1, \ldots, N^{t}-1\right\},
$$

где число $N=2,3, \ldots$ является параметром модели. Этот параметр назовем $n a p a м e m-$ ром деления. Будем считать, что процесс $\widetilde{Y}_{t}$ является марковским. По определению положим, что $\widetilde{Y}_{0}=\{0\}$. Тогда в моменты времени $t>0$ процесс определяется по условной вероятности перехода

$$
\begin{aligned}
P_{t}(B, A) & =\mathbf{P}\left\{\widetilde{Y}_{t+1}=A \mid \widetilde{Y}_{t}=B\right\}= \\
& = \begin{cases}0, & B \not \subset \mathfrak{A}_{t}, \\
(1-q)^{|A \backslash B|} q^{\left|\mathfrak{A}_{t+1} \backslash A\right|}, & B \subset A \text { либо } B \not \subset A\end{cases}
\end{aligned}
$$

Таким образом, переход от $\widetilde{Y}_{t}$ к $\widetilde{Y}_{t+1}$ состоит в добавлении к $\widetilde{Y}_{t}$ случайно выбранных с вероятностью $(1-q)$ из множества $\mathfrak{A}_{t+1} \backslash \widetilde{Y}_{t}$ точек - новых границ фрагментов. Этим правилом перехода случайный процесс $\left\{\widetilde{Y}_{t}\right\}$ и, следовательно, процесс $\left\{\widetilde{X}_{t}=\left\{\tilde{\sigma}_{i} ; i=\right.\right.$ $1,2, \ldots, \tilde{\nu}(t)\} ; t=0,1,2, \ldots\}$ полностью определены, равно как и случайный процесс $\{\tilde{\nu}(t)\}$, представляюший собой число связных компонент реализации $\widetilde{X}(t)$, причем $N$ и $q$ - свободные параметры модели. Непосредственно из этого определения следует лемма 1.

ЛЕмма 1. Имеет место свойство нормировки

$$
\sum_{A: B \subset A \subset \mathfrak{A}_{t+1}} P_{t}(B, A)=1 .
$$


ДокАЗАТЕЛЬСТво.

$$
\begin{aligned}
\sum_{A: B \subset A \subset \mathfrak{A}_{t+1}} P_{t}(B, A) & =\sum_{A: B \subset A \subset \mathfrak{A}_{t+1}}(1-q)^{|A \backslash B|} q^{\left|\mathfrak{A}_{t+1} \backslash A\right|}= \\
& =\sum_{A \subset \mathfrak{A}_{t+1}}(1-q)^{|A|} q^{\left|\left(\mathfrak{A}_{t+1} \backslash B\right) \backslash A\right|}=1^{\left|\mathfrak{A}_{t+1} \backslash B\right|},
\end{aligned}
$$

что и требовалось доказать.

Следуюшее утверждение показывает, что в каждый момент $t \in \mathbb{N}$ реализации процесса $\widetilde{Y}_{t}$ представляют собой неоднородные последовательности независимых испытаний на множестве $\mathfrak{A}_{t}$.

\section{Теорема 1. Имеет место формула}

$$
\mathbf{P}\left\{\widetilde{Y}_{t}=A\right\}=\prod_{s=1}^{t}\left(1-q^{t-s+1}\right)^{\left|A \cap\left(\mathfrak{A}_{s} \backslash \mathfrak{A}_{s-1}\right)\right|} q^{(t-s+1)\left|\mathfrak{A}_{s} \backslash\left(\mathfrak{A}_{s-1} \cup A\right)\right|} .
$$

ДокАЗАТЕЛЬСтво. Осуществим индукцию по $t$. При $t=1$ формула (9) верна в силу $(8)$ и условия $\widetilde{Y}_{0}=\{0\}$. Индукционный шаг осушествим на основе соглашения о марковости процесса $\left\{\widetilde{Y}_{t}\right\}$,

$$
\mathbf{P}\left\{\tilde{Y}_{t+1}=A\right\}=\sum_{B: B \subset A \cap \mathfrak{A}_{t}} P_{t}(B, A) \mathbf{P}\left\{\widetilde{Y}_{t}=B\right\} .
$$

Представим $A=\bigcup_{s=1}^{t+1} A_{s}$, где $A_{s}=A \cap\left(\mathfrak{A}_{s} \backslash \mathfrak{A}_{s-1}\right)$, и аналогично $B=\bigcup_{s=1}^{t} B_{s}$, где $B_{s} \subset A_{s}$. Преобразуем правую часть в (10) на основе формул (8) и (9),

$$
\begin{aligned}
\mathbf{P} & \left\{\tilde{Y}_{t+1}=A\right\}= \\
= & \sum_{B: B \subset A \cap \mathfrak{A}_{t}}(1-q)^{|A \backslash B|} q^{\left|\mathfrak{A}_{t+1} \backslash A\right|} \prod_{s=1}^{t}\left(1-q^{t-s+1}\right)^{\left|B \cap\left(\mathfrak{A}_{s} \backslash \mathfrak{A}_{s-1}\right)\right|} q^{(t-s+1)\left|\mathfrak{A}_{s} \backslash\left(\mathfrak{A}_{s-1} \cup B\right)\right|}= \\
= & (1-q)^{\left|\mathfrak{A}_{t+1}\right|} q^{\left|\mathfrak{A}_{t+1} \backslash \mathfrak{A}_{t} \backslash A_{t+1}\right|} R\left(A_{1}, \ldots, A_{t+1}\right), \\
R= & \sum_{\substack{B_{s} \subset A_{s} \\
s=1, \ldots, t}}\left[\prod_{s=1}^{t}(1-q)^{\left|A_{s} \backslash B_{s}\right|} q^{\left|\mathfrak{A}_{s} \backslash \mathfrak{A}_{s-1} \backslash A_{s}\right|}\right] \times \\
& \times \prod_{s=1}^{t}\left(1-q^{t-s+1}\right)^{\left|B_{s}\right|} q^{(t-s+1)\left|\mathfrak{A}_{s} \backslash\left(\mathfrak{A}_{s-1} \cup B_{s}\right)\right|}= \\
= & \prod_{s=1}^{t}\left[\sum_{B_{s} \subset A_{s}}(1-q)^{\left|A_{s} \backslash B_{s}\right|} q^{\left|\mathfrak{A}_{s} \backslash \mathfrak{A}_{s-1} \backslash A_{s}\right|}\left(1-q^{t-s+1}\right)^{\left|B_{s}\right|} q^{(t-s+1)\left|\mathfrak{A}_{s} \backslash\left(\mathfrak{A}_{s-1} \cup B_{s}\right)\right|}\right] . \quad \text { (11) }
\end{aligned}
$$

Так как $\mathfrak{A}_{s} \backslash\left(\mathfrak{A}_{s-1} \cup B_{s}\right)=\left(\mathfrak{A}_{s} \backslash\left(\mathfrak{A}_{s-1} \cup A_{s}\right)\right) \cup\left(A_{s} \backslash B_{s}\right)$, сумма в квадратных скобках в формуле (11) представляется в виде

$$
q^{(t-s+2)\left|\mathfrak{A}_{s} \backslash\left(\mathfrak{A}_{s-1} \cup A_{s}\right)\right|} \sum_{B_{s} \subset A_{s}}\left(1-q^{t-s+1}\right)^{\left|B_{s}\right|}\left[(1-q) q^{t-s+1}\right]^{\left|A_{s} \backslash B_{s}\right|}=
$$




$$
\begin{aligned}
& =q^{(t-s+2)\left|\mathfrak{A}_{s} \backslash\left(\mathfrak{A}_{s-1} \cup A_{s}\right)\right|}\left[(1-q) q^{t-s+1}+1-q^{t-s+1}\right]^{\left|A_{s}\right|}= \\
& =q^{(t-s+2)\left|\mathfrak{A}_{s} \backslash\left(\mathfrak{A}_{s-1} \cup A_{s}\right)\right|}\left(1-q^{t-s+2}\right)^{\left|A_{s}\right|} .
\end{aligned}
$$

Подставляя это выражение в (11), получаем формулу (9) при $t+1$. Теорема 1 доказана.

Пусть $\left\{\tilde{y}_{i}(t) \in \tilde{Y}_{t}\right\}$ - точки из $\widetilde{Y}_{t}$, упорядоченные по возрастанию, а фрагментами являются связные компоненты индуцированного случайного процесса $\left\{\widetilde{X}_{t}\right\}$, построенного по формуле (7). Фрагменты описываются семейством интервалов $\left\{\left(\tilde{y}_{i-1}(t), \tilde{y}_{i}(t)\right)=\right.$ $\left.\tilde{\sigma}_{i}(t) ; i=1, \ldots, \tilde{\nu}(t)\right\}$. С целью изучения статистики связных компонент введем бесконечномерный случайный процесс с траекториями

$$
\tilde{\nu}_{l}(t)=\operatorname{Card}\left\{i:\left(\tilde{y}_{i}(t)-\tilde{y}_{i-1}(t)\right) N^{t}=l\right\}, \quad l=1,2, \ldots,
$$

где числа $N^{t}\left(\tilde{y}_{i}(t)-\tilde{y}_{i-1}(t)\right)=\tilde{r}_{i}(t)-\tilde{r}_{i-1}(t)$ будем называть перенормированными размерами фрагментов $\left(\tilde{y}_{i-1}(t), \tilde{y}_{i}(t)\right)=\tilde{\sigma}_{i}(t)$, а случайную величину $\tilde{\nu}_{l}(t)$ - числом фрагментов длины $l$ (в единицах $N^{-t}$ ) в момент $t$.

При каждом $t$ случайные последовательности по $l$ при фиксированном $t$ с вероятностью 1 имеют только конечный набор отличных от нуля компонент. При этом

$$
\sum_{l=1}^{N^{t}} l \tilde{\nu}_{l}(t)=N^{t}, \quad \tilde{\nu}_{l}(t)=0, \text { если } l>N^{t} .
$$

Для исследования процесса $\left\{\left\{\tilde{\nu}_{l}(t)\right\} ; t \in \mathbb{N}_{0}\right\}$ рассмотрим последовательность независимых испытаний $\{\tilde{\xi}(n)\}$, введенную в предыдущем разделе. Пусть $\pi\left\{k_{l} ; l \in \mathbb{N} \mid s\right\}-$ вероятность появления в наборе точек деления с номерами $n=1, \ldots, s-1$ из этой последовательности ровно $k_{l}, l=1,2, \ldots$, фрагментов длины $l$. Здесь под фрагментами мы понимаем наборы $\tilde{\rho}_{i}=\left(\tilde{r}_{i}+1, \ldots, \tilde{r}_{i}+l=\tilde{r}_{i+1}\right)$, где $i=1,2, \ldots, k$ и $k=\sum_{l=1}^{\infty} k_{l}-$ число всех фрагментов, а $\tilde{r}_{i}-$ их начальные точки, причем $\tilde{r}_{1}=0$. Для этой вероятности имеется явная формула

$$
\pi\left\{k_{l} ; l \in \mathbb{N} \mid s\right\}=q^{s-k}(1-q)^{k-1} C\left\{k_{l} ; l \in \mathbb{N} \mid s\right\} \quad \text { при условии } s=\sum_{l=1}^{\infty} l k_{l},
$$

где комбинаторный множитель

$$
C\left\{k_{l} ; l \in \mathbb{N} \mid s\right\}=\frac{k !}{\prod_{l=1}^{\infty} k_{l} !}
$$

Если же $s \neq \sum_{l=1}^{\infty} l k_{l}$, то $\pi\left\{k_{l} ; l \in \mathbb{N} \mid s\right\}=0$. Легко понять, что происхождение этого комбинаторного множителя связано с числом всех возможных размешений фрагментов $\tilde{\rho}_{i}$, отождествленных согласно своим размерам $l$, т.е. с повторениями по $k_{l}$ элементов в каждом классе эквивалентности. Именно посредством учёта всех таких размешений пересчитываются все реализации последовательности $\{\tilde{\xi}(n) ; n=1,2, \ldots, s-1\}$, характеризуемые набором “чисел заполнения" $\left\{k_{l} ; l=1,2, \ldots\right\}$. 
Введем теперь вероятности $\mathbf{P}\left\{\tilde{\nu}_{l}(t)=n_{l} ; l \in \mathbb{N}\right\} \equiv P_{t}\left(n_{l} ; l \in \mathbb{N}\right)$ - характеристики процесса $\left\{\tilde{\nu}_{l}(t) ; t=0,1,2, \ldots\right\}$. Они имеют вид

$$
P_{t}\left(n_{l} ; l \in \mathbb{N}\right)=\sum_{A: A \subset \mathfrak{A}_{t}, \rho_{l}(A, t)=n_{l}} \mathbf{P}\left\{\tilde{Y}_{t}=A\right\},
$$

где $\rho_{l}(A, t)$ - число фрагментов длины $l$ в последовательности фрагментов $\left\{\tilde{\rho}_{i}\right\}$, построенных по указанному вьше принципу, конечные точки которых в наборе $(1, \ldots, s)$, $s=N^{t}$ совпадают с точками $N^{t} A$, т.е. $\tilde{\rho}_{i}=N^{t} \tilde{\sigma}_{i}$.

Докажем, что случайный процесс $\left\{\tilde{\nu}_{l}(t) ; t=0,1,2, \ldots\right\}$ со значениями в виде наборов чисел заполнения является марковским, т.е. он полностью определяется условными вероятностями перехода, вычисляемыми согласно следуюшей формуле:

$$
\begin{aligned}
\mathbf{P}\left\{\tilde{\nu}_{l}(t+1)\right. & \left.=n_{l} ; l \in \mathbb{N} \mid \tilde{\nu}_{s}(t)=m_{s} ; s \in \mathbb{N}\right\}= \\
& =\sum_{\left\{k_{l}^{(s, i)}\right\}}^{m_{s}} \prod_{s=1}^{\infty} \prod_{i=1}^{m_{s}} \pi\left\{k_{l}^{(s, i)} ; l \in \mathbb{N} \mid N s\right\} .
\end{aligned}
$$

Здесь использована введенная выше функция $\pi\left\{k_{l} ; l \in \mathbb{N} \mid s\right\}$, посредством которой описывается вероятность развала фрагмента длиной $s$ в реализации процесса $\left\{\widetilde{Y}_{t}\right\}$ после его растяжения в $N$ раз на набор, состоящий из $k_{l}, l \in \mathbb{N}$, фрагментов с длиной $l$ при переходе от $t$ к $t+1$. Эти вероятности по построению процесса $\left\{\widetilde{Y}_{t}\right\}$ не зависят от $t$. Суммирование в формуле (15) распространяется на все наборы $\left\{k_{l}^{(s, i)} ; l \in \mathbb{N}, s \in \mathbb{N}, i=1, \ldots, m_{s}\right\}$, для которых выполняются связи

$$
\sum_{s=1}^{N^{t}} \sum_{i=1}^{m_{s}} k_{l}^{(s, i)}=n_{l} \quad \text { и } \quad \sum_{l=1}^{\infty} l k_{l}^{(s, i)}=N s, \quad i=1, \ldots, m_{s} ; \quad s \in \mathbb{N} .
$$

Теорема 2. Вероятности $P_{t}\left(n_{l} ; l \in \mathbb{N}\right)$ подчинены әволюиионному уравнению

$$
\begin{aligned}
& P_{t+1}\left(n_{l} ; l \in \mathbb{N}\right)= \\
& \quad=\sum_{\left\{m_{s} ; s \in \mathbb{N}\right\}} \mathbf{P}\left\{\tilde{\nu}_{l}(t+1)=n_{l} ; l \in \mathbb{N} \mid \tilde{\nu}_{s}(t)=m_{s} ; s \in \mathbb{N}\right\} P_{t}\left(m_{s} ; s \in \mathbb{N}\right),
\end{aligned}
$$

суммирование в әтой и в аналогичных формулах ниже выполняется по всем возможным значениям наборов чисел заполнения $m_{s}=0,1,2, \ldots$ с $s=1,2, \ldots$.

ДокАЗАТЕЛЬСтво. Доказательство осуществим индукцией по $t$. При $t=1$ имеем

$$
\sum_{\substack{A: 0 \in A \mathcal{C L}_{1} \\ \rho_{l}(A, 1)=n_{l}}} P\left(\mathfrak{A}_{0}, A\right)=\sum_{\substack{A: 0 \in A \in \mathcal{A}_{1} \\ \rho_{l}(A, 1)=n_{l}}}(1-q)^{\left|A \backslash \mathfrak{A}_{0}\right|} q^{\left|\mathfrak{A}_{1} \backslash A\right|}=(1-q)^{n-1} q^{N-n} C\left\{n_{l} ; l \in \mathbb{N} \mid N\right\},
$$

где $n=\sum_{l=1}^{\infty} n_{l}$. Осушествим теперь индукционный шаг от $t$ к $t+1$. Пусть $A \subset \mathfrak{A}_{t+1}$, $\rho_{l}(A, t+1)=n_{l}$ и $P_{t}(B, A) \neq 0$, т.е. $B \subset A, B \subset \mathfrak{A}_{t}$. Пусть каждый фрагмент $\tilde{\rho}_{i}$, $i=1, \ldots, m_{s}$, имеюшийся во множестве $B$ к моменту $t$ и обладающий длиной $s$, порождает $k_{l}^{(s, i)}$ фрагментов длиной $l$ при переходе ко множеству $A$ в момент $t+1$. Тогда 
с необходимостью выполняется вся совокупность соотношений (16). Обратно, если для множества $B \subset \mathfrak{A}_{t}$ выполняется равенство $\rho_{s}(B, t)=m_{s}$ и если выполняются связи $(16)$, то для множества $B$ найдется такое множество $A \subset \mathfrak{A}_{t+1}$, для которого $\rho_{l}(A, t+1)=n_{l}$, $P_{t}(B, A) \neq 0$. Следовательно, справедливо следуюшее преобразование оператора суммирования $\sum_{A: A \subset \mathfrak{A}_{t+1}, \rho_{l}(A, t+1)=n_{l}}$ при применении его к равенству $(10)$ :

$$
\begin{aligned}
& \sum_{A: A \subset \mathfrak{A}_{t+1}, \rho_{l}(A, t+1)=n_{l}} \mathbf{P}\left\{\tilde{Y}_{t+1}=A\right\}= \\
& =\sum_{m_{s} \in \mathbb{N}}\left[\sum_{B: B \subset \mathfrak{A}_{t}, \rho_{s}(B, t)=m_{s}} \mathbf{P}\left\{\tilde{Y}_{t}=B\right\}\left(\sum_{\left\{k_{l}^{(s, i)}\right\}}^{m_{s}} P_{t}(B, A)\right)\right] .
\end{aligned}
$$

“Лишние" слагаемые при суммировании по $m_{s}, s \in \mathbb{N}$, т.е. такие, для которых не вьполняются условия (16), автоматически выпадают из-за обрашения в нуль условной вероятности перехода. Заметим теперь, что условная вероятность (8) может быть представлена в виде

$$
\prod_{s=1}^{\infty} \prod_{i=1}^{m_{s}} \pi\left\{k_{l}^{(s, i)} ; l \in \mathbb{N} \mid N s\right\}
$$

если выполняются соотношения (16). Так как при учете этого факта внутренняя сумма в правой части равенства не зависит от переменной $B$, то можно выполнить суммирование по этой переменной, воспользовавшись формулой (14). Поступая аналогичнос левой частью равенства, получим уравнение (17). Теорема 2 доказана.

Структура условных вероятностей (15) указьвает на тот факт, что случайный процесс $\left\{\tilde{\nu}_{l}(t)\right\}$ является ветвящимся [12]. Поэтому, как и для любого ветвящегося марковского процесса, из уравнения (17) следует эволюционное уравнение для одноточечной производяшей функции

$$
\Psi_{t}\{Z\} \equiv \sum_{\left\{n_{l} ; l \in \mathbb{N}\right\}}\left(\prod_{s=1}^{\infty} z_{s}^{n_{s}}\right) P_{t}\left(n_{l} ; l \in \mathbb{N}\right) .
$$

Эта функция зависит от бесконечных наборов $Z=\left(z_{1}, z_{2}, \ldots\right)$, где $z_{i} \in[0,1], i=$ $1,2,3, \ldots$, таких, что только конечные наборы компонент в $Z$ отличны от 1 .

ТЕОремА 3. Функиия $\Psi_{t}\{Z\}$ удовлетворяет уравнению

$$
\Psi_{t+1}\{Z\}=\Psi_{t}\{Q\{Z\}\}
$$

где отображсние $\mathrm{Q}:[0,1]^{\mathbb{N}} \rightarrow[0,1]^{\mathbb{N}}$ определено формулой

$$
(\mathrm{Q}\{Z\})_{s}=\sum_{\left\{k_{l} ; l \in \mathbb{N}\right\}} \pi\left\{k_{l} ; l \in \mathbb{N} \mid N s\right\} \prod_{l=1}^{\infty} z_{l}^{k_{l}}
$$


ДоКАзАТЕЛЬСТво. Используя выражения (15) и (16), запишем

$$
\begin{aligned}
& \Psi_{t+1}\{Z\}= \\
& =\sum_{\left\{n_{l} ; l \in \mathbb{Z}\right\}}\left(\prod_{l=1}^{\infty} z_{l}^{n_{l}}\right) \sum_{\left\{m_{s} ; s \in \mathbb{Z}\right\}} P_{t}\left\{m_{s} ; s \in \mathbb{N}\right\} \sum_{\left\{k_{l}^{(s, i)}\right\}}^{m_{s}} \prod_{s=1}^{\infty} \prod_{i=1}^{m_{s}} \pi\left\{k_{l}^{(s, i)} ; l \in \mathbb{N} \mid N s\right\} .
\end{aligned}
$$

Переставляя суммирования и воспользовавшись уравнением (18), правую часть последнего равенства преобразуем к виду

$$
\begin{aligned}
& \sum_{\left\{m_{s} ; s \in \mathbb{Z}\right\}} P_{t}\left\{m_{s} ; s \in \mathbb{N}\right\} \sum_{\left\{n_{l} ; l \in \mathbb{Z}\right\}} \sum_{\left\{k_{l}^{(s, i)}\right\}}^{m_{s}} \prod_{s=1}^{\infty} \prod_{i=1}^{m_{s}} \pi\left\{k_{l}^{(s, i)} ; l \in \mathbb{N} \mid N s\right\} \prod_{l=1}^{\infty} z_{l}^{k_{l}^{(s, i)}}= \\
& =\sum_{\left\{m_{s} ; s \in \mathbb{Z}\right\}} P_{t}\left\{m_{s} ; s \in \mathbb{N}\right\} \times \\
& \quad \times \quad \sum_{\left\{k_{l}^{(s, i)} ; l \in \mathbb{Z}, s \in \mathbb{N}, i=1, \ldots, m_{s}\right\}} \prod_{s=1}^{\infty} \prod_{i=1}^{m_{s}} \pi\left\{k_{l}^{(s, i)} ; l \in \mathbb{N} \mid N s\right\} \prod_{l=1}^{\infty} z_{l}^{k_{l}^{(s, i)}}= \\
& =\sum_{\left\{m_{s} ; s \in \mathbb{Z}\right\}} P_{t}\left\{m_{s} ; s \in \mathbb{N}\right\} \prod_{s=1}^{\infty} \prod_{i=1}^{m_{s}} \sum_{\left\{k_{l}^{(s, i)} ; l \in \mathbb{Z}\right\}} \pi\left\{k_{l}^{(s, i)} ; l \in \mathbb{N} \mid N s\right\} \prod_{l=1}^{\infty} z_{l}^{k_{l}^{(s, i)}}= \\
& =\sum_{\left\{m_{s} ; s \in \mathbb{Z}\right\}} P_{t}\left\{m_{s} ; s \in \mathbb{N}\right\} \prod_{s=1}^{\infty} \prod_{i=1}^{m_{s}}(\mathrm{Q}\{Z\})_{s}=\Psi_{t}\{\mathrm{Q}\{Z\}\} .
\end{aligned}
$$

Теорема 3 доказана.

Введем одноточечные моменты случайного процесса $\left\{\tilde{\nu}_{l}(t)\right\}$,

$$
\left\langle\tilde{\nu}_{l}(t)\right\rangle=\sum_{n=1}^{\infty} n \mathbf{P}\left\{\tilde{\nu}_{l}(t)=n\right\} .
$$

Они вычисляются на основе производящей функции $\Psi\{Z\}$,

$$
\left\langle\tilde{\nu}_{l}(t)\right\rangle=\left(\frac{\partial}{\partial z_{l}} \Psi\{Z\}\right)_{Z \equiv 1} .
$$

Используя уравнения (19), (20), находим, что, как и для любого ветвящегося процесса, имеется линейное преобразование, связьвающее средние значения $\left\langle\tilde{\nu}_{l}(t)\right\rangle$ в соседних временных точках,

$$
\left\langle\tilde{\nu}_{l}(t+1)\right\rangle=\sum_{m=1}^{\infty} \mathcal{M}_{l m}\left\langle\tilde{\nu}_{m}(t)\right\rangle,
$$

где

$$
\mathcal{M}_{l m}=\left(\frac{\partial(\mathrm{Q}\{Z\})_{m}}{\partial z_{l}}\right)_{Z \equiv 1}=\sum_{n=1}^{\infty} n \pi_{l}(n \mid m)
$$

и $\pi_{l}(n \mid m)$ - вероятность такого развала фрагмента с размером $m$ за один эволюционный шаг (т.е. растяжение в $N$ раз и последуюшее дробление), в результате которого образуется ровно $n$ фрагментов размера $l$. 


\section{5. АНАЛИЗ КОМБИНАТОРНЫХ КОЭФФИЦИЕНТОВ}

Из определения вероятности $\pi_{l}(n \mid m)$ следует, что

$$
\pi_{l}(n \mid m)=\sum_{\left\{k_{s} ; s \in \mathbb{N}\right\}} \delta_{n k_{l}} \pi\left\{k_{s} ; s \in \mathbb{N} \mid N m\right\}
$$

Это согласуется с соотношениями (20), (22). Воспользовавшись формулами (12), (13), находим

$$
\begin{aligned}
\pi_{l}(n \mid m)= & \sum_{k=n}^{N m-n(l-1)}(1-q)^{k-1} q^{N m-k} C_{l}^{(k)}(n \mid N m), \\
C_{l}^{(k)}(n \mid N m)= & \sum_{\left\{k_{s} ; s \in \mathbb{N}\right\}} \delta_{n k_{l}} C\left\{k_{s} ; s \in \mathbb{N} \mid N m\right\} \times \\
& \times \delta\left(N m-\sum_{s=1}^{\infty} s k_{s}\right) \delta\left(k-\sum_{s=1}^{\infty} k_{s}\right),
\end{aligned}
$$

где $\delta(j) \equiv \delta_{0 j}$ при $j \in \mathbb{Z}$ и $C_{l}^{(k)}(n \mid N m)$ - число всех разбиений $\left(\rho_{1}, \ldots, \rho_{k}\right)$ упорядоченного множества $\{1,2, \ldots, N m\}$ на связные фрагменты с размерами $l_{i}$, т.е. на последовательности $\rho_{i}=\left(r_{i}+1, \ldots, r_{i}+l_{i}\right), i=1,2, \ldots, k$, где $r_{i+1}=r_{i}+l_{i}, r_{i} \in\{0,1, \ldots, N m-1\}$ при $i=1,2, \ldots, k$ и $r_{1}=0, r_{k}+l_{k}=N m$. Причем в этих разбиениях присутствует ровно $n$ фрагментов размера $l$.

В этом разделе вычислим производящую функцию для комбинаторных коэффициентов (24), которая определяется как

$$
V(z)=\sum_{m=(l-1) n+k}^{\infty} z^{m} C_{l}^{(k)}(n \mid N m) .
$$

$\mathrm{C}$ этой целью рассмотрим банахово пространство $\mathbb{L}(\alpha), 0<\alpha<1$, последовательностей $u=\left\{u_{n} ; n=0,1,2, \ldots\right\}$, для которых конечна норма

$$
\left\|u_{l}\right\|_{\alpha}=\sup _{m \in \mathbb{N}} \alpha^{-m}\left|u_{m}\right|<\infty
$$

Для каждой из таких последовательностей сушествует производяшая функция

$$
U(z)=\sum_{n=1}^{\infty} z^{n} u_{n}, \quad|z|<\alpha .
$$

Введем на пространстве $\mathbb{L}(\alpha)$ операцию умножения. Для двух последовательностей $f=$ $\left\{f_{n}\right\}$ и $g=\left\{g_{n}\right\}$ определим последовательность $h \equiv f * g$ формулой для ее компонент

$$
h_{n}=(f * g)_{n}=\sum_{m=0}^{n} f_{m} g_{n-m}, \quad n \in \mathbb{N}_{0} .
$$


Тогда производящая функция $H(z)$ последовательности $h$ является произведением производящих функций последовательностей $f$ и $g, F(z) G(z)=H(z)$. Обозначим $n$-ю степень последовательности $f$ из построенной алгебры символом $f_{*}^{n}$, где $f_{*}^{n}=f_{*}^{n-1} * f$, $n \in \mathbb{N} ;\left(f_{*}^{0}\right)_{n}=\delta_{0 n}$.

Введем теперь для данного фиксированного $l \in \mathbb{N}$ последовательности $a=\left\{\delta_{l n} ; n=\right.$ $0,1,2, \ldots\}$ и $b=\left\{1-\delta_{l n}-\delta_{0 n} ; n=0,1,2, \ldots\right\}$. Их производяшие функции есть соответственно $A(z)=z^{l}$ и $B(z)=z /(1-z)-z^{l}$.

Лемма 2. Имеет место формула

$$
C_{l}^{(k)}(n \mid s)=\left(\begin{array}{l}
k \\
n
\end{array}\right)\left(a_{*}^{n} * b_{*}^{k-n}\right)_{s}
$$

ДокАЗАТЕЛЬство. Семейство $\mathfrak{C}_{l}^{(k+1)}(n \mid s)$ всех упорядоченных разбиений $\left(\rho_{1}, \ldots\right.$, $\left.\rho_{k+1}\right)$ множества $\{1,2, \ldots, s\}$, содержаших $n$ фрагментов длины $l$, распадается на объединение непересекающихся семейств разбиений

$$
\left(\bigcup_{\substack{j=1 \\ j \neq l)}}^{s-n(l-1)-k} \mathfrak{C}_{l}^{(k)}(n \mid s-j)\right) \cup \mathfrak{C}_{l}^{(k)}(n-1 \mid s-l)
$$

где каждое из семейств, входящих в первое объединение, состоит из разбиений $\left(\rho_{1}, \ldots\right.$, $\left.\rho_{k+1}\right)$ таких, что длина последнего фрагмента $\rho_{k+1}$ в них равна $j, j \neq l$. Все такие разбиения образуются однозначным образом из разбиений $\left(\rho_{1}, \ldots, \rho_{k}\right)$ множества $\{1, \ldots, s-j\}$ присоединением фрагмента $\rho_{k+1}$. Тогда число элементов в каждом семействе равно соответственно $C_{l}^{(k)}(n \mid s-j)$. При этом максимально возможное значение $j$ определяется тем, что кроме $\rho_{k+1}$ имеется $n$ фрагментов длины $l$ и $(k-n)$ фрагментов единичной длины.

Разбиения из семейства $\mathfrak{C}_{l}^{(k)}(n-1 \mid s-l)$ таковы, что последний фрагмент в них имеет длину $l$. По той же причине, что и выше, число всех элементов в этом семействе равно $C_{l}^{(k)}(n-1 \mid s-l)$. Тогда справедлива формула

$$
C_{l}^{(k+1)}(n \mid s)=\sum_{\substack{j=1 \\ j \neq l)}}^{s-n(l-1)-k} C_{l}^{(k)}(n \mid s-j)+C_{l}^{(k)}(n-1 \mid s-l)
$$

Положив формально, что $C_{l}^{(k)}(n \mid s)=0$ при $s<k+(l-1) n$, и введя последовательности $\left\{(S(k, n))_{s} \equiv C_{l}^{(k)}(n \mid s) ; s=0,1,2, \ldots\right\}, k \geqslant n=0,1,2, \ldots$, последнюю формулу можно записать в виде

$$
S(k+1, n)=S(k, n) * b+S(k, n-1) * a .
$$

Разностное уравнение (28) по двум переменным $k$ и $n$ с $k \geqslant n$ дополняется двумя краевыми условиями. Во-первых, $S(k, k)=a_{*}^{k}$, так как при $k=n$ все фрагменты, 
на которые расщепляется фрагмент с длиной $s$, имеют длину $l$ и поэтому существует только одно разбиение, если $s=k l$, а в противном случае разбиения отсутствуют, $C_{l}^{(k)}(k \mid s)=\delta(s-k l)=\left(a_{*}^{k}\right)_{s}$. Во-вторых, $S(k, 0)=b_{*}^{k}$, т.е. $C_{l}^{(k)}(0 \mid s)=\left(b_{*}^{k}\right)_{s}$.

Индукцией по $k=1,2, \ldots$ устанавливается, что уравнение (28) имеет единственное решение с заданными краевыми условиями. С другой стороны, $S(k, n)=\left(\begin{array}{l}k \\ n\end{array}\right) a_{*}^{n} * b_{*}^{k-n}$ удовлетворяет уравнению и краевым условиям. Лемма 2 доказана.

СлЕДСтвиЕ. Производящая функиия для комбинаторных коэффициентов $C_{l}^{(k)}(n \mid s)$ имеет вид

$$
S(k, n ; z)=\sum_{s=1}^{\infty} z^{s} C_{l}^{(k)}(n \mid s)=\left(\begin{array}{l}
k \\
n
\end{array}\right) z^{n l} B^{k-n}(z) .
$$

Лемма 3. Имеет место оценка

$$
V(z) \leqslant \frac{z^{\frac{1-N}{N}}}{N} S\left(k, n ; z^{\frac{1}{N}}\right), \quad 1 \geqslant z>0 .
$$

ДокАЗАтЕльство. Очевидно, что $C_{l}^{(k)}(n \mid N m)<C_{l}^{(k)}(n \mid N m+j)$ с $m \in \mathbb{N}$, $j=1, \ldots, N-1$. Кроме того, $z^{m} \leqslant z^{(1-N) / N}\left(z^{1 / N}\right)^{N m+j}$. Используя эти неравенства, функция $V(z)$ согласно $(25)$ оценивается как

$$
V(z)<\frac{z^{\frac{1-N}{N}}}{N} \sum_{m=1}^{\infty} \sum_{j=0}^{N-1}\left(z^{\frac{1}{N}}\right)^{N m+j} C_{l}^{(k)}(n \mid N m+j)=\frac{z^{\frac{1-N}{N}}}{N} S\left(k, n ; z^{\frac{1}{N}}\right) .
$$

Здесь мы использовали условие $C_{l}^{(k)}(n \mid N m)=0$ при $N m<n(l-1)+k$. Лемма 3 доказана.

\section{6. КАЧЕСТВЕННОЕ ИССЛЕДОВАНИЕ ПРОЦЕССА ФРАГМЕНТАЦИИ}

Введем для процесса фрагментации $\left\{X_{t}\right\}$ распределение $p_{l}(t)$ размеров фрагментов в момент времени $t \in \mathbb{N}_{0}$ согласно формуле

$$
\left\{p_{l}(t)=\frac{\left\langle\tilde{\nu}_{l}(t)\right\rangle}{\langle\tilde{\nu}(t)\rangle} ; l \in \mathbb{N}\right\} \equiv p(t) .
$$

Каждое такое распределение является неотрицательным элементом пространства $\mathbb{L}_{1}$ суммируемых последовательностей. Наша цель - установить сушествование единственной предельной точки для последовательностей $p(t), \lim _{t \rightarrow \infty} p(t)=p$ в топологии пространства $\mathbb{L}(\alpha)$, если $p(0) \in \mathbb{L}(\alpha)$ и $q<\alpha^{(N-1) / N}$ и, в частности, при $p_{l}(0)=\delta_{1 l}$, когда $p_{l}(t)=0$ при достаточно больших $l$. Эволюция распределения $p(t)=\left\{p_{l}(t)\right\}$ при этом определяется формулой, вытекаюшей из (21),

$$
p_{l}(t+1)=\frac{\sum_{m=1}^{\infty} \mathcal{M}_{l m} p_{m}(t)}{\sum_{l, m=1}^{\infty} \mathcal{M}_{l m} p_{m}(t)} \equiv \frac{(\mathrm{M} p(t))_{l}}{(\mathbf{1}, \mathrm{M} p(t))}, \quad \mathbf{1}=(1,1, \ldots) .
$$


При этом вследствие закона больших чисел $p_{l}(t) \sim \tilde{\nu}_{l}(t) / \tilde{\nu}(t)$.

Для достижения этой цели необходимо показать, что траектория $p_{l}(t)$ не выходит за пределы пространства $\mathbb{L}(\alpha)$ с выбранным значением $\alpha$ и в $\mathbb{L}(\alpha)$ сушествует единственная притягивающая неподвижная точка. Докажем вспомогательное утверждение.

TEOPEмA 4. Oператор $\mathrm{M}$ ограничен в каждом из пространств $\mathbb{L}(\alpha)$ при $\alpha^{(N-1) / N}>q(\alpha<1)$ и для его матрицы выполняется свойство суммируемости,

$$
\sum_{l, m=1}^{\infty} \alpha^{m-l} \mathcal{M}_{l m}<\infty
$$

ДокАЗАТЕльство. Пусть $\left\{u_{l}\right\} \in \mathbb{L}(\alpha)$. Тогда

$$
(\mathrm{M} u)_{l} \leqslant\|u\|_{\alpha} \sum_{m=1}^{\infty} \mathcal{M}_{l m} \alpha^{m} .
$$

Согласно формулам (23) и (30) ряд, стояший в правой части неравенства (34), оценивается величиной

$$
\begin{aligned}
\sum_{m=1}^{\infty} \sum_{n=1}^{\infty} n \alpha^{m} \pi_{l}(n \mid m) & =q^{-1} \sum_{n=1}^{\infty} n \sum_{k=n}^{\infty}\left[q^{-1}(1-q)\right]^{k} V\left(\alpha q^{N}\right) \leqslant \\
& \leqslant q^{-1} \frac{\left(\alpha^{\frac{1}{N}} q\right)^{1-N}}{N} \sum_{n=1}^{\infty} n \sum_{k=n}^{\infty}\left[q^{-1}(1-q)\right]^{k} S\left(k, n ; \alpha^{\frac{1}{N}} q\right)
\end{aligned}
$$

Внутренняя сумма в (35) вычисляется явно после подстановки (29), что дает

$$
\sum_{k=n}^{\infty}\left[q^{-1}(1-q)\right]^{k} S\left(k, n ; \alpha^{\frac{1}{N}} q\right)=(1-\zeta)^{-1} \eta^{n},
$$

где $\zeta=q^{-1}(1-q) B\left(q \alpha^{1 / N}\right), \eta=\alpha^{l / N} q^{l-1}(1-q)(1-\zeta)^{-1}$. Сходимость ряда при $\alpha \leqslant 1$ вытекает из элементарной оценки $B(z)<z(1-z)^{-1}, 0<z<1$. Подставляя полученное выражение в (35) и суммируя степенной ряд, получим

$$
\sum_{m=1}^{\infty} \mathcal{M}_{l m} \alpha^{m} \leqslant \eta q^{-1} \frac{\left(\alpha^{\frac{1}{N}} q\right)^{1-N}}{N}(1-\zeta)^{-1}(1-\eta)^{-2} .
$$

При этом $\eta<1$, так как это неравенство эквивалентно очевидному неравенству $B\left(q \alpha^{1 / N}\right)<q(1-q)^{-1}-\left(q \alpha^{1 / N}\right)^{l}$ при $\alpha<1$. Далее, учитывая $(34)$, получаем

$$
\|\mathrm{M} u\|_{\alpha} \leqslant \mathrm{const}\|u\|_{\alpha} \sup _{l \in \mathbb{N}} \alpha^{-l} \eta(1-\eta)^{-2}
$$

где const не зависит от $l$. Верхняя грань конечна, если $q<\alpha^{(N-1) / N}$. При этих же условиях из (36) вытекает (33). Теорема 4 доказана.

Докажем теперь основной результат работы. 
Теорема 5. Для любого $q<1$ существует единственное распределение $p_{l}$ по размерам фрагментов со строго положительными компонентами и такое, что при начальном распределении $\left\{p_{l}(0)\right\} \in \mathbb{L}(\alpha)$ с $q<\alpha^{(N-1) / N}<1$ прочесс фрагментации $\left\{\tilde{X}_{t}\right\}$ порождает последовательность распределений $\left\{p_{l}(t) ; t=0,1,2, \ldots\right\} \in$ $\mathbb{L}(\alpha)$, определенных формулой $(31)$, сходящуюся $к$ распределению $p_{l} \in \mathbb{L}(\alpha)$.

ДокАЗАТЕЛЬСтво. Если $p(0) \in \mathbb{L}(\alpha)$, то, не ограничивая общности, всегда можно считать, что $\alpha^{(N-1) / N}>q$. В этих условиях достаточно доказать сходимость последовательности распределений $p(t)$ при любом таком начальном распределении $p(0)$.

Ввиду свойства суммируемости $(33)$ матрица $\alpha^{m-l} \mathcal{M}_{l m}$ порождает компактный оператор в пространстве $\mathbb{L}_{1}$. Поэтому оператор $\mathrm{M}$ является компактным в пространстве $\mathbb{L}(\alpha)$.

Введем оператор $D=M /\|M\|_{\alpha}$. Он компактен в пространстве $\mathbb{L}(\alpha)$. Компактность гарантирует точечность спектра и конечномерность инвариантных относительно действия $\mathrm{D}$ подпространств в $\mathbb{L}(\alpha)$. Следовательно, оператор $\mathrm{D}$ имеет только конечньй набор собственных значений $\{\lambda\}$, модуль которых $\max \{|\lambda|\}=1$. При этом соответствуюшие инвариантные подпространства конечномерны.

C другой стороны, матрица оператора D имеет неотрицательные элементы. Кроме того, из определения $\mathcal{M}_{l m}$ следует, что эти матричные элементы обрашаются в нуль, только если $l>m N$. В этом и только в этом случае $\pi_{l}(n \mid m)=0$ при всех $n$. В частности, $\mathcal{M}_{l l} \neq 0$. Это гарантирует неразложимость матрицы $\mathcal{M}_{l m}$ и соответственно матрицы $\alpha^{m-l} \mathcal{M}_{l m}$ [13], [14]. Неразложимость означает отсутствие такого разбиения $\mathbb{N}=K \cup L, K \cap L=\varnothing$, для которого $\mathcal{M}_{k l}=0$ при $k \in K, l \in L$ и $l \in K, k \in L$.

Для неотрицательных неразложимых матриц справедливо обобщение известной теоремы Фробениуса (см. приложение). Применение этой теоремы к матрице оператора $D$ в сочетании с компактностью этого оператора позволяет заключить, что на единичной окружности имеется только одна точка спектра - простое собственное число равное 1. У него имеется собственный вектор со строго положительными компонентами $\left\{p_{l}\right\}, \sum_{l=1}^{\infty} p_{l}=1$. Все остальные собственные значения оператора $\mathbf{D}$ по модулю строго меньше 1. Это позволяет заключить, что для любого вектора $p(0)$, обладающего свойством $\|p(0)\|_{\alpha}<\infty$ с $\alpha>0$ и достаточно близким к 1 , выполняется в $\mathbb{L}(\alpha)$-топологии соотношение

$$
\lim _{t \rightarrow \infty}\left(\mathrm{D}^{t} p\right)(0)=p_{l}
$$

Наконец, индукцией по $t=1,2, \ldots$ легко доказывается на основании формулы $(31)$, что

$$
p(t)=\frac{\mathrm{D}^{t} p(0)}{\left(\mathbf{1}, \mathrm{D}^{t} p(0)\right)}, \quad \mathbf{1}=(1,1, \ldots) .
$$

Переходя к пределу по $t \rightarrow \infty$, получаем требуемое утверждение. Теорема 5 доказана.

\section{7. ЗАКЛЮЧЕНИЕ}

Предложенный в данной работе подход к описанию процессов фрагментации на основе построения стохастической геометрии среды, подвергающейся процессам эрозии, хотя и 
усложняет их изучение, однако позволяет более реалистически учесть детали этих процессов, имеющие чисто геометрическое происхождение. Реализация данного подхода в одномерном случае привела к новому типу предельных законов распределения с экспоненциальными хвостами. Эти законы не являются универсальными, что очень важно с точки зрения возможности экспериментально изучать приводящие к ним динамические воздействия.

\section{ПРИЛОЖЕНИЕ}

Дадим формулировку утверждения, использованного нами при доказательстве теоремы 5. Доказательство этого утверждения в приведенной формулировке осушествляется переносом соответствуюшего доказательства с конечномерного случая (по поводу теорем Перрона и Фробениуса для конечных матриц см., например, [13], [15]).

УТвеРЖДЕНИЕ. Если $\mathcal{M}_{k l} \geqslant 0$ - неразложимая матрица, $\mathcal{M}_{l l} \neq 0$, и если точки спектра оператора $\mathrm{M}$ в пространстве $\mathbb{L}_{1}$, порождаемого ею в каноническом базисе $e^{(k)}=\left\{\delta_{n k} ; n \in \mathbb{N}\right\}, \quad k \in \mathbb{N}$, которые лежат на окружности с радиусом $\|\mathrm{M}\|$, составляют часть его точечного спектра, то их множество состоит из одного простого собственного числа $\lambda=\|\mathrm{M}\|$. При этом соответствующий собственный вектор можсно выбрать так, чтобы все его компоненты были строго положительны.

Благодарности. Работа выполнена при поддержке гранта "Университеты России" УР-991702.

\section{Список литературы}

[1] А. Н. Колмогоров. ДАН СССР. 1941. Т. 31. № 2. С. 99.

[2] А. Ф. Филиппов. Теория вероят. и ее применен. 1961. Т. 6. № 3. С. 299.

[3] A. Charlesby. Proc. Roy. Soc. (London). Ser. A. 1954. V. 224. P. 120.

[4] R. M. Ziff, E. D. McGrady. J. Phys. A. 1985. V. 18. P. 3027.

[5] Р. З. Сагдеев, А. В. Тур, В. В. Яновский. ДАН СССР. 1987. Т. 294. № 5. С. 1105.

[6] R. M. Ziff,. J. Phys. A. 1991. V. 24. P. 2421.

[7] P. L. Krapivsky, E. Ben-Naim. Phys. Rev. E. 1994. V. 50. P. 3502.

[8] R. Balescu. Equilibrium and Nonequilibrium Statistical Mechanics. New York: John Wiley \& Sons, 1975.

[9] Yu. P. Virchenko, O. I. Sheremet. Доповіді НАН Украі́ни. 2000. № 8. Р. 82-86.

[10] И. И. Гихман, А. В. Скороход. Введение в теорию случайных процессов. М.: Наука, 1977.

[11] G. Matheron. Random Sets and Integral Geometry. New York: John Wiley \& Sons, 1975.

[12] Б. А. Севастьянов. Ветвящиеся процессы. М.: Наука, 1971.

[13] Ф. Р. Гантмахер. Теория матриц. М.: Наука, 1966.

[14] Э. Нуммелин. Общие неприводимые цепи Маркова и неотрицательные операторы. М.: Мир, 1989.

[15] Р. Беллман. введение в теорию матриц. М.: Наука, 1976.

Поступила в редакцию 10.VIII.2000 г., после доработки 6.III.2001 г.

2 Теоретическая и математическая физика, т. 128, № 2, 2001 г. 